\title{
Copolymerizable photoinitiators based on benzophenones and their influence on shrinkage of photoreactive acrylic PSAs
}

\author{
Zbigniew Czech*, Agnieszka Kowalczyk, Dominika Sowa, \\ Jagoda Kowalska, Paulina Ragańska \\ Institute of Chemical Organic Technology, West Pomeranian University of Technology, Szczecin, \\ K. Pulaskiego 10, 70-322 Szczecin, Poland \\ *E-mail address: psa_czech@wp.pl
}

\begin{abstract}
This manuscript describes a novel class of copolymerizable benzophenone photoinitiators and their influence on viscosity and molecular mass of acrylic pressure-sensitive adhesives (PSAs) during radical solvent polymerization and shrinkage of acrylic PSAs coated on PVC film and crosslinked using UV radiation.
\end{abstract}

Keywords: copolymerizable benzophenone photoinitiators; UV-crosslinking; acrylic; pressuresensitive adhesive (PSA); shrinkage; viscosity; molecular mass

\section{INTRODUCTION}

Industrial applications of photoreactive UV crosslinkable materials have grown tremendously over the past decade. An entire industry has evolved based on the existence of chemicals, which respond to UV radiation. The growing success of UV crosslinking technologies in a wide variety of industrial products and processes can be attributed to the productivity and environmental benefits that result from such technologies [1].

UV technology is well established in the industry and allows the production of a wide range of UV-crosslinkable pressure-sensitive adhesives with interesting performance. The balance between adhesive and cohesive strengths within the crosslinked coatings is critical for the performance of the UV crosslinked PSA [2-4]. The advantages of UV-crosslinking over thermal crosslinking include rapid network formation (in a fraction of a second by using high intensity light sources) and the utilization of heat-sensitive substrates.

UV radiation is widely used in industrial processes. The UV equipment like UV-lamps and UV-lasers emits radiation in the UV region between 200 and $400 \mathrm{~nm}$ of the electromagnetic spectrum [5]. The remaining required investment is the modification of the coating machine with UV-lamps. The crosslinking with UV can be done directly after the application or after passing a cooling zone. UV-lamps are categorized as low, medium and high pressure UV-lamps [1].

Mercury lamps (low, medium, high pressure) are used with powers between 80 and 120 W/cm which includes UV stations with six and more UV lamps with power between 120 and $260 \mathrm{~W} / \mathrm{cm}$. These types of UV-lamp possess the right wave-length bands in their emission 
spectrum as needed for an efficient UV-crosslinking of photoreactive PSAs. The UV-A wavelengths, responsible for UV crosslinking are in the range between 320 to $400 \mathrm{~nm}$. Spectral emissions of common UV-A radiators for this application also contain other wavelengths for example IR components [5].

Increasing attention is given to a new class of unsaturated copolymerizable photoinitiators and their polymerisation with others acrylic monomers and their influence on properties of synthesized PSA containing these mentioned photoinitiators such as shrinkage after UV crosslinking [6].

During UV radiation the intermolecular benzophenone $\mathrm{H}$-abstractors are excited and react with the neighbouring $\mathrm{C}$-H positions of polymer side-chains. UV crosslinkable acrylic PSAs possess excellent oxidation resistance which allows working without inert gas atmosphere [7-10, 12].

\section{EXPERIMENTAL}

\section{1. Materials}

The following experiments were performed to study the influence of kind and amount of copolymerizable benzophenone photoinitiators and UV-crosslinking time on shrinkage of acrylic pressure-sensitive adhesives. The basic PSAs were synthesized with between 62 to $64.9 \%$ butyl acrylate (BA), $30 \%$ methyl acrylate (MA), $5 \%$ acrylic acid (AA) and between 0,1 to $3 \%$ unsaturated benzophenone photoinitiators during radical polymerisation in ethyl acetate [11]. The solvent-borne acrylic PSAs were synthesized with solid content on $50 \mathrm{wt} \%$ using of $0.1 \mathrm{wt} \%$. on radical initiator AIBN concentration. The dosage time during polymerisation process was $3 \mathrm{~h}$ and the post-reaction time $5 \mathrm{~h}$.

Butyl acrylate (BA), methyl acrylate (MA), acrylic acid (AA), ethyl acetate and AIBN (2,2`-azo-diisobutyonitrile) are purchased from BASF (Germany). The investigated copolymerizable benzophenone photoinitiators with different organic spacer: 4-acryloyloxy benzophenone (ABP) (Figure 1), 4-(2-acryloyloxy-ethoxy) benzophenone (AEBP) (Figure 2), 4-(2-acryl-oyloxy-butoxy) benzophenone (ABBP) (Figure 3) and 4-(2-acryloyloxy-hexoxy) benzophenone (AHBP) (Figure 4) were synthesized by Chemitec (Germany).

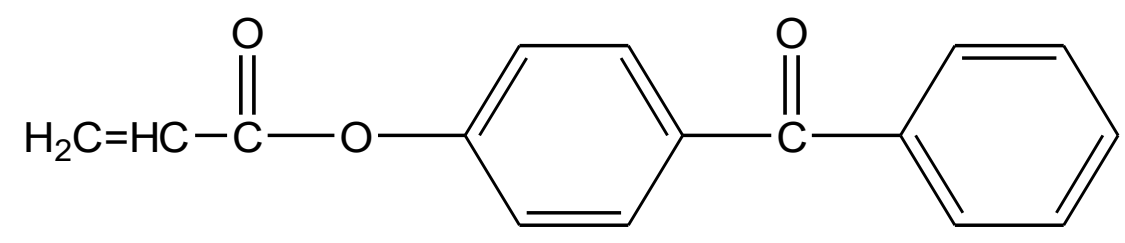

Figure 1. 4-acryloyloxy benzophenone (ABP).

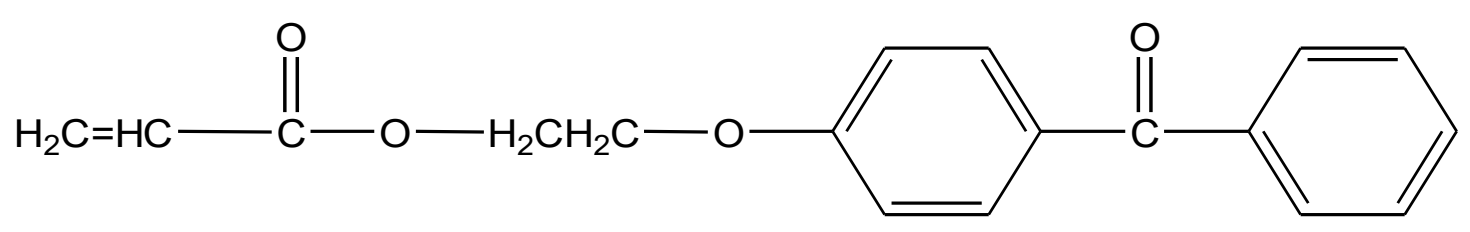

Figure 2. 4-(2-acryloyloxy-ethoxy) benzophenone (AEBP). 


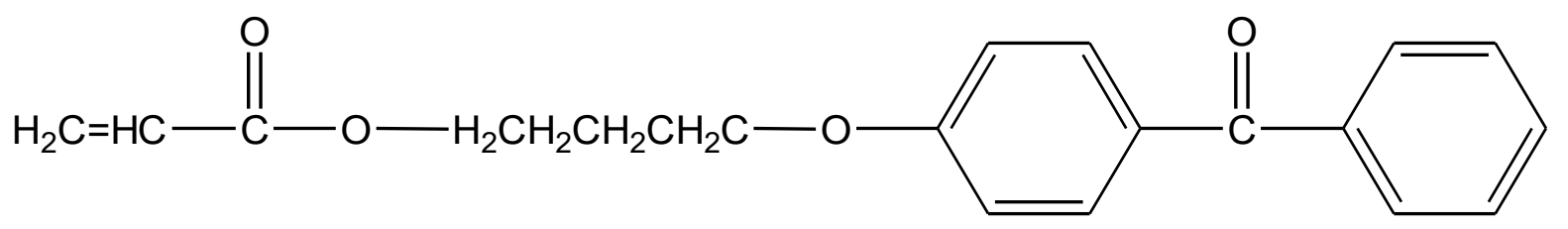

Figure 3. 4-(2-acryloyloxy-butoxy) benzophenone (ABBP).

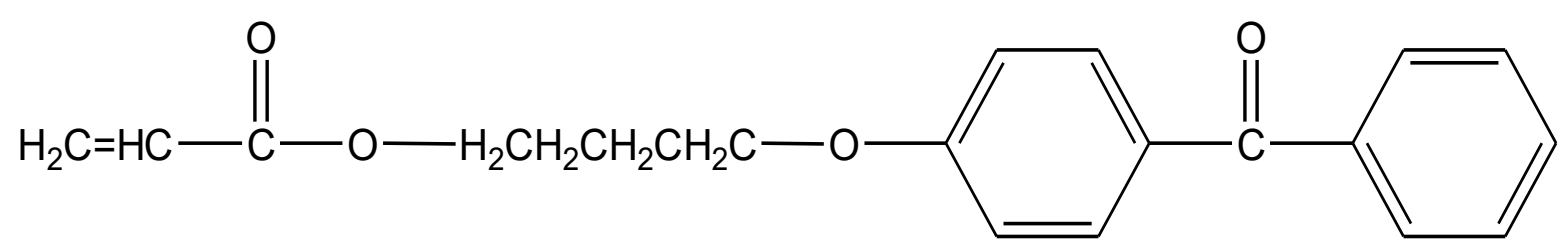

Figure 4. 4-(2-acryloyloxy-hexoxy) benzophenone (AHBP).

\section{2. Measurements}

The viscosity of the investigated solvent-borne acrylics pressure-sensitive adhesives was determined with a Rheomat RM 189 from Rheometric Scientific, with spindle No 3 at 23 ${ }^{\circ} \mathrm{C}$ and the molecular weight studies were performed in tetrahydrofurane with a liquid chromatograph LaChrom system: RI Detector L-7490 and LaChrom UV Detector L-7400 from Merck-Hitachi, equipped with a PLgel $10^{6} \AA$ A column from Hewlett-Packard.

The shrinkage presents the percentage or millimeter change of dimensions of the PVC film covered with PSA after crosslinking and attached to the glass after keeping it 4 weeks at temperature of $60{ }^{\circ} \mathrm{C}$. With shrinkage greater than $0.5 \%$ or greater than $0.5 \mathrm{~mm}$ other properties were neglected.

\section{RESULTS AND DISCUSSION}

Influence of copolymerizable photoinitiators based on benzophenones on viscosity and molecular mass of synthesized acrylic pressure-sensitive adhesives (PSAs)

The composition of synthesized acrylic pressure-sensitive adhesives containing copolymerizable benzophenone photoinitiators, their viscosity and molecular mass were illustrated in Tables 1-4. 
Table 1. Important properties of synthesized PSA containing 4-acryloyloxybenzophenone (ABP).

\begin{tabular}{|c|c|c|c|c|c|c|}
\hline \multirow{2}{*}{$\begin{array}{c}\text { PSA } \\
\text { No }\end{array}$} & \multicolumn{4}{|c|}{ Kind of monomeres [wt \%] } & \multirow{2}{*}{$\begin{array}{c}\eta \\
{[\mathbf{P a} \cdot \mathbf{s}]}\end{array}$} & \multirow{2}{*}{$\stackrel{\mathbf{M}_{W}}{\text { [dalton] }}$} \\
\hline & $\mathrm{BA}$ & MA & $\mathrm{AA}$ & $\mathrm{ABP}$ & & \\
\hline 1 & 65.0 & 30 & 5 & 0 & 3.0 & 804000 \\
\hline 2 & 64.9 & 30 & 5 & 0.1 & 3.6 & 846000 \\
\hline 3 & 64.5 & 30 & 5 & 0.5 & 3.9 & 865000 \\
\hline 4 & 64.0 & 30 & 5 & 1.0 & 4.3 & 922000 \\
\hline 5 & 63.5 & 30 & 5 & 1.5 & 4.8 & 932000 \\
\hline 6 & 63.0 & 30 & 5 & 2.0 & 5.5 & 941000 \\
\hline 7 & 62.5 & 30 & 5 & 2.5 & 6.7 & 962000 \\
\hline 8 & 62.0 & 30 & 5 & 3.0 & 9.6 & 996000 \\
\hline
\end{tabular}

Table 2. Important properties of synthesized PSA containing 4-(2-acryloyloxy-ethoxy)benzophenone) (AEBP).

\begin{tabular}{|c|c|c|c|c|c|c|}
\hline \multirow{2}{*}{$\begin{array}{c}\text { PSA } \\
\text { No }\end{array}$} & \multicolumn{4}{|c|}{ Kind of monomeres [wt \%] } & \multirow{2}{*}{$\begin{array}{c}\boldsymbol{\eta} \\
{[\mathbf{P a} \cdot \mathbf{s}]}\end{array}$} & $\begin{array}{c}\mathbf{M}_{\mathbf{W}} \\
\text { [dalton] }\end{array}$ \\
\cline { 2 - 5 } & BA & MA & AA & AEBP & & 742000 \\
9 & 64,9 & 30 & 5 & 0.1 & 2.5 & 755000 \\
10 & 64,5 & 30 & 5 & 0.5 & 2.6 & 778000 \\
11 & 64,0 & 30 & 5 & 1.0 & 3.0 & 791000 \\
12 & 63,5 & 30 & 5 & 1.5 & 3.2 & 832000 \\
13 & 63,0 & 30 & 5 & 2.0 & 3.8 & 888000 \\
14 & 62,5 & 30 & 5 & 2.5 & 4.1 & 922000 \\
15 & 62,0 & 30 & 5 & 3.0 & 5.9 & \\
\hline
\end{tabular}

Table 3. Important properties of synthesized PSA containing 4-(2-acryloyloxy-butoxy) benzophenone (ABBP).

\begin{tabular}{|c|c|c|c|c|c|c|}
\hline \multirow{2}{*}{$\begin{array}{l}\text { PSA } \\
\text { No }\end{array}$} & \multicolumn{4}{|c|}{ Kind of monomeres [wt \%] } & \multirow{2}{*}{$\begin{array}{c}\eta \\
{[\mathrm{Pa} \cdot \mathrm{s}]}\end{array}$} & \multirow{2}{*}{$\underset{\text { [dalton] }}{\mathbf{M}_{\mathbf{W}}}$} \\
\hline & $\mathrm{BA}$ & MA & $\mathrm{AA}$ & ABBP & & \\
\hline 16 & 64,9 & 30 & 5 & 0.1 & 2.0 & 667000 \\
\hline 17 & 64,5 & 30 & 5 & 0.5 & 2.2 & 681000 \\
\hline 18 & 64,0 & 30 & 5 & 1.0 & 2.6 & 703000 \\
\hline 19 & 63,5 & 30 & 5 & 1.5 & 3.0 & 724000 \\
\hline 20 & 63,0 & 30 & 5 & 2.0 & 3.5 & 763000 \\
\hline 21 & 62,5 & 30 & 5 & 2.5 & 3.9 & 815000 \\
\hline 22 & 62,0 & 30 & 5 & 3.0 & 4.9 & 863000 \\
\hline
\end{tabular}


Table 4. Important properties of synthesized PSA containing 4-(2-acryloyloxy-hexoxy) benzophenone (AHBP).

\begin{tabular}{|c|c|c|c|c|c|c|}
\hline \multirow{2}{*}{$\begin{array}{l}\text { PSA } \\
\text { No }\end{array}$} & \multicolumn{4}{|c|}{ Kind of monomeres [wt \%] } & \multirow{2}{*}{$\begin{array}{c}\eta \\
{[\mathbf{P a} \cdot \mathbf{s}]}\end{array}$} & \multirow{2}{*}{$\stackrel{\mathbf{M}_{W}}{\text { [dalton] }}$} \\
\hline & $\mathrm{BA}$ & MA & AA & AHBP & & \\
\hline 23 & 64,9 & 30 & 5 & 0,1 & 1.4 & 514000 \\
\hline 24 & 64,5 & 30 & 5 & 0,5 & 1.6 & 544000 \\
\hline 25 & 64,0 & 30 & 5 & 1,0 & 2.0 & 569000 \\
\hline 26 & 63,5 & 30 & 5 & 1,5 & 2.3 & 610000 \\
\hline 27 & 63,0 & 30 & 5 & 2,0 & 2.8 & 643000 \\
\hline 28 & 62,5 & 30 & 5 & 2,5 & 3.6 & 721000 \\
\hline 29 & 62,0 & 30 & 5 & 3,0 & 4,5 & 829000 \\
\hline
\end{tabular}

The influence of the copolymerizable benzophenone photoinitiators on viscosity and molecular mass of synthesized acrylic PSAs are described in the graphs shown in Figs. 5-6.

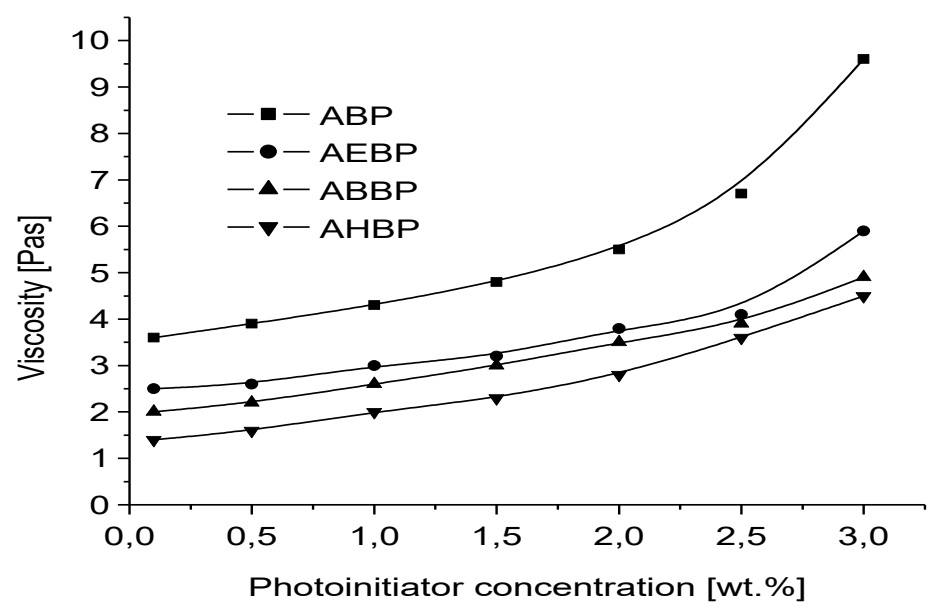

Figure 5. Effect of photoinitiator kind and concentration on PSA viscosity.

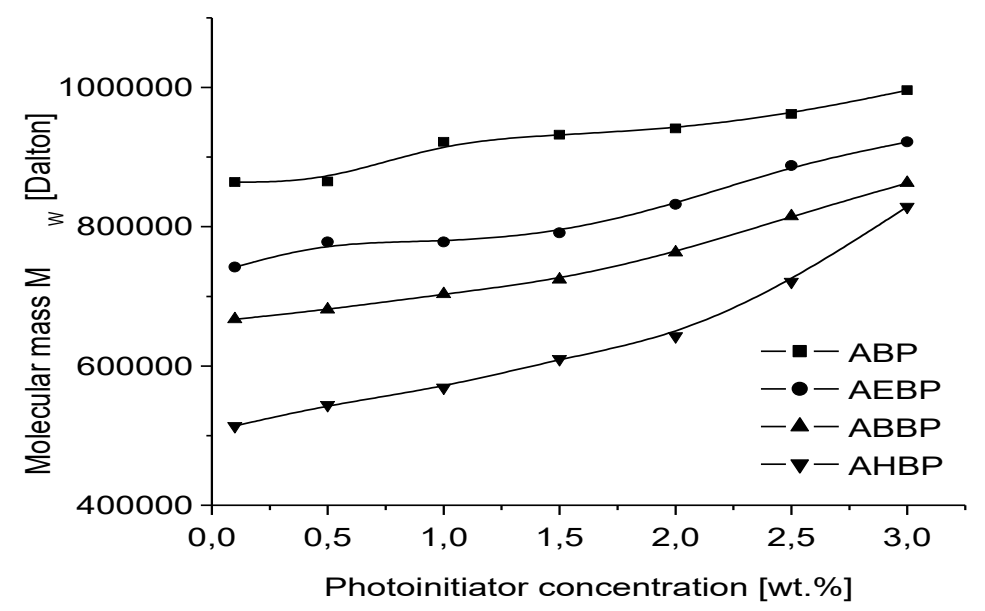

Figure 6. Effect of photoinitiator kind and concentration on PSA molecular mass. 
The following conclusions can be inferred from conducted experimental results:

- the increase of the unsaturated benzophenone photoinitiator amount corresponds directly proportional with the increase of viscosity of synthesized acrylic pressuresensitive adhesives,

- in general, the increase of unsaturated benzophenone photoinitiator content increases the molecular mass of synthesized acrylic PSA,

- from the investigated unsaturated benzophenone photoinitiators, the best results for viscosity and molecular mass ameliorating were given by benzophenone photoinitiators with shorter organic spacer between copolymerizable double bond and benzophenone chromophoric structure (ABP, AEBP).

\section{Influence of unsaturated benzophenone photoinitiators kind, amount and crosslinking time on shrinkage}

It was the aim to examine the influence of tested copolymerizable benzophenone pfotoinitiators on shrinkage of synthesized acrylic pressure-sensitive adhesive after UVcrosslinking using UV-lamp (UV Spot from Dr. Hoenle UV-Technology-Germany).

The influence of copolymerizable benzophenone pfotoinitiators kind and concentration on shrinkage of UV-crosslinked acrylic PSAs by the same crosslinking time is shown in Figure 7. In Figure 8 is presented the influence of crosslinking time on shrinkage of acrylic PSA by the same selected concentration of unsaturated benzophenones.

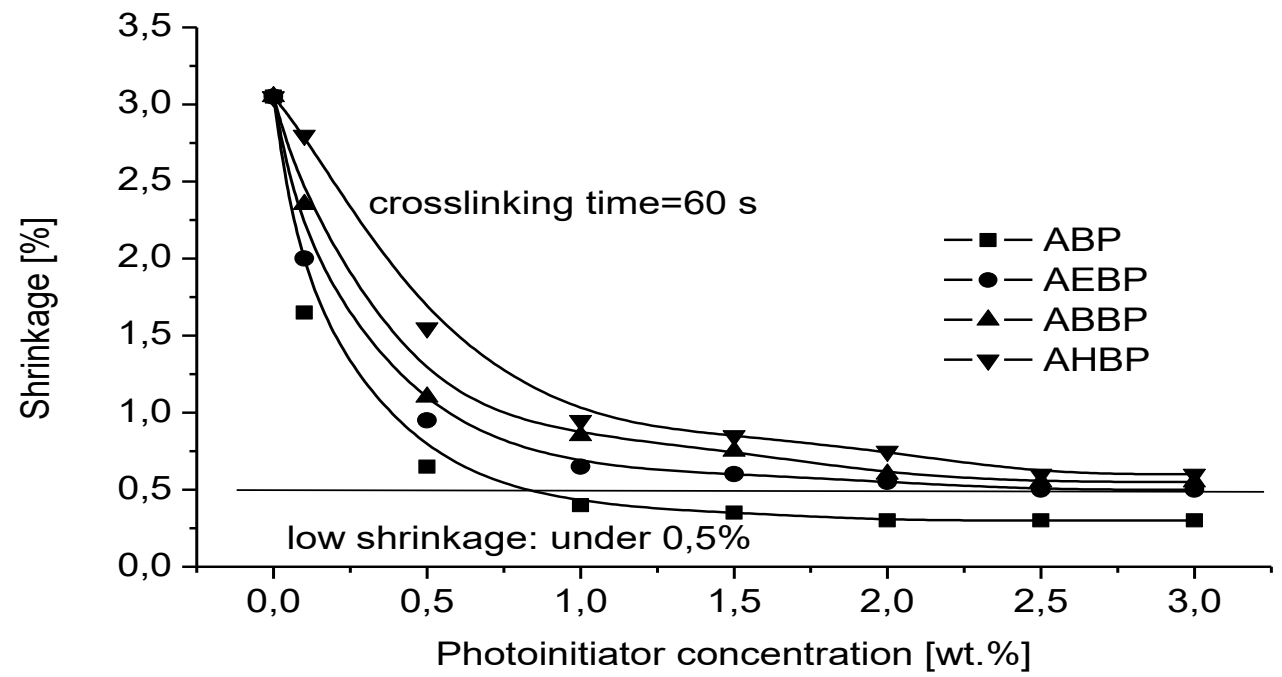

Figure 7. Shrinkage dependent on photoinitiator concentration.

General, it can be said that the use of copolymerizable benzophenones such as photoinitiators offers an interesting alternative to the other methods of reducing the shrinkage of pressure-sensitive adhesive coated on PVC films. The best results with respect to shrinkage are observed in the case of 4-acryloyloxy benzophenone (ABP). With ca. about $0.75 \mathrm{wt} \%$ ABP the shrinkage of PSA was lower than $0.5 \%$. Low or very low shrinkage $<0.5 \%$ is the best parameter of synthesized acrylic pressure-sensitive adhesive with excellent performance for PVC surfaces. 


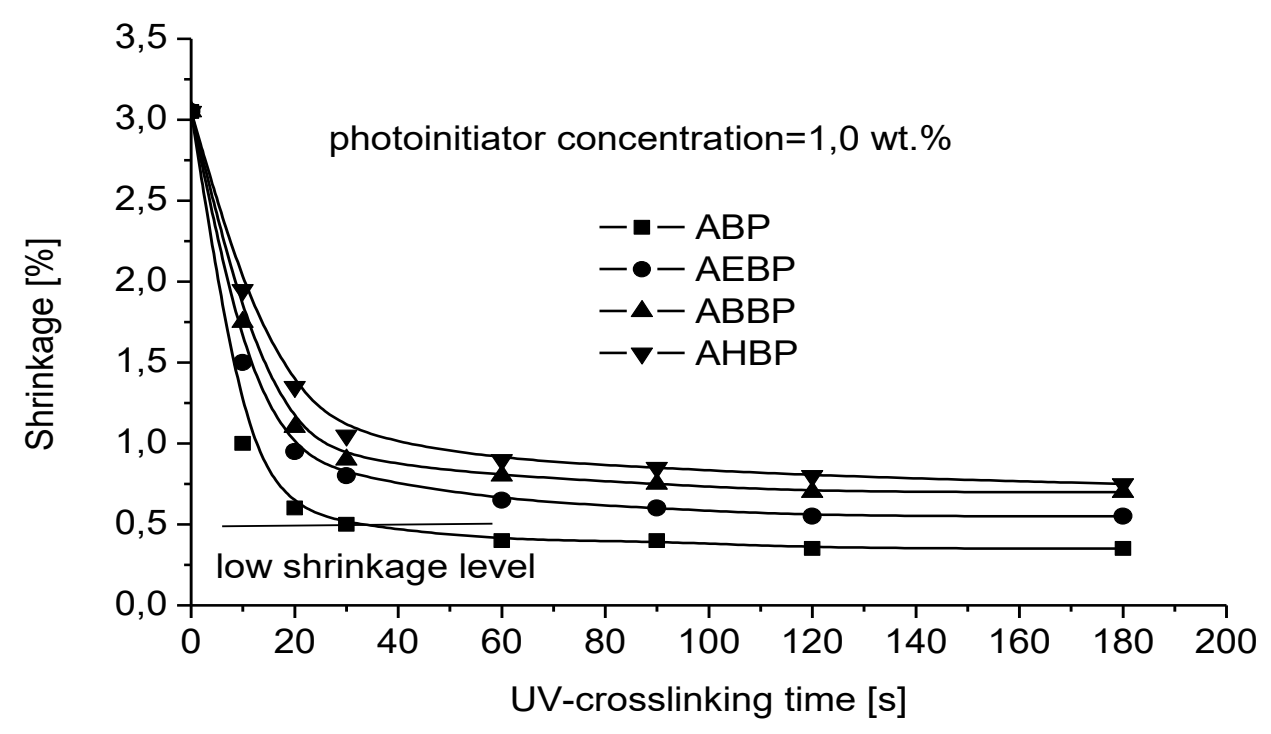

Figure 8. Shrinkage dependent on UV-crosslinking time.

Increasing the UV-crosslinking time of acrylic pressure-sensitive adhesives leads clearly to less shrinkage. The best shrinkage performance was observed after UV-crosslinking for crosslinking time greater than $30 \mathrm{~s}$. The use of several unsaturated benzophenones as photoinitiators showed the most efficient results for 4-acryloyloxy benzophenone (ABP).

\section{CONCLUSIONS}

From the evaluation of the conducted experiments discussed in this manuscript, it can be concluded that:

- From the investigated copolymerozable benzophenone photoinitiators, the excellent result of low shrinkage was observed by using of 4-acryloyloxy benzophenone (ABP).

- Increasing the amount of copolymerizable benzophenone photoinitiator increases viscosity and molecular mass of synthesized acrylic pressure-sensitive adhesives

- Only the use of 4-acryloyloxy benzophenone (ABP) as copolymerizable photoinitiator with concentration greater than 0.75 wt. \% quarantined a relatively low shrinkage

- The best shrinkage resistance simultaneous with longer UV-crosslinking time. After 30 second UV-crosslinking time the shrinkage level for acrylic PSAs containing unsaturated photoinitiator 4-acryloyloxy benzophenone (ABP) was very low, exactly $0.45 \mathrm{~mm}$ and was with it acceptable for sign and marking industry. 


\section{References}

[1] Z. Czech, Crosslinking of Acrylic PSA, Publisher Szczecin University of Technology, 1999.

[2] R. Milker, Z. Czech, Polimery 5 (1987) 182-188.

[3] R. Milker, Z. Czech, Journal of Applied Polymer Science 86 (2002) 1354-1360.

[4] Z. Czech, Polimery 10 (2006) 754-757.

[5] Z. Czech, A. Kowalczyk, J. Kabatc, J. Świderska, European Polymer Journal 48 (2012) 1446-1454.

[6] I. Benedek, Developments in Crosslinking of Solvent-Based Acrylics, Taylor \& Francis a CRC Press Book 2006.

[7] Z. Czech, A. Butwin, J. Kabatc, J. Świderska, Polymer Bulletin 69 (2012) 71-80.

[8] Z. Czech, A. Malec, Polish Journal of Chemical Technology 2 (2006) 5-8.

[9] Z. Czech, W. Blum, F. Herrmann, patent WO 94/14 853 (1993).

[10] Z. Czech, A. Butwin, J. Kabatc, J. Świderska, L. Shao, Y. Bai, Polymer Bulletin 68, (2012) 441-452.

[11] Zbigniew Czech, Agnieszka Kowalczyk, Dominika Sowa, International Letters of Chemistry, Physics and Astronomy 1 (2013) 63-69.

[12] Zbigniew Czech, Dominika Sowa, Jagoda Kowalska, International Letters of Chemistry, Physics and Astronomy 3 (2013) 21-28. 